\begin{tabular}{|l|l|l|l|l|l|}
\hline MUNIBE Antropologia-Arkeologia & $n^{\circ} 67$ & $363-375$ & DONOSTIA & 2016 & ISSN $1132-2217 \bullet$ elSSN 2172-4555 \\
\hline
\end{tabular}

\title{
San Adrian: un nuevo yacimiento de la Edad del Bronce en el norte de la península Ibérica
}

\author{
San Adrian: Brontze Aroko aztarnategi berria Iberiar penintsulako iparraldean \\ San Adrian: a new site of the Bronze Age in the north of the Iberian peninsula
}

PALABRAS CLAVES: Estructuras de habitación; Hábitat de montaña; Estratigrafía; Cerámica; Fauna; Industria lítica; Industria ósea; Paleoambiente; Dataciones.

GAKO-HITZAK: Bizileku-egiturak; Mendiko habitata; Estratigrafia; Zeramika; Fauna; Harri-industria; Hezur-industria; Paleo-ingurumena; Datazioak. KEY WORDS: Domestic structures; Mountain habitat; Stratigraphy; Caeramic; Fauna; Lithic tools; Bone industry; Palaeoenvironment; Radiocarbon dates.

\begin{abstract}
Jesús TAPIA(1), Miriam CUBAS(2,1), Manuel CEBERIO(1), Alfredo MORAZA ${ }^{(1)}$, Juantxo AGIRRE-MAULEON(1), Euken ALONSO ${ }^{(4,1)}$, Esteban ÁLVAREZ-FERNÁNDEZ(5), Pablo ARESO ${ }^{(1)}$, ÁNGEL ARMENDARIZ ${ }^{(3,1)}$, Pedro CASTAÑOS ${ }^{(1)}$, Jone CASTAÑOS ${ }^{(7)}$, Francisco ETXEBERRIA ${ }^{(1)}$, Joseba GARMENDIA ${ }^{(1)}$, Lourdes HERRASTI(1), María José IRIARTE CHIAPUSSO(6), Daniel PÉREZ ${ }^{(1)}$, Ana URIZ(1), Lydia ZAPATA(†) ${ }^{(7)}$
\end{abstract}

\section{RESUMEN}

La investigación sobre la Edad del Bronce en la región cantábrica se ha centrado tradicionalmente en el estudio de objetos de prestigio y de contextos funerarios, dando lugar a un estado de la cuestión con importantes lagunas acerca del poblamiento, las actividades cotidianas y las estrategias de subsistencia de los grupos humanos durante este período.

En este artículo presentamos las evidencias recuperadas en el yacimiento de San Adrian (Sierra de Aizkorri, Gipuzkoa), cuya excavación ha permitido reconstruir aspectos relativos a los modos de vida, la base económica, la cultura material y el contexto medioambiental de una serie de ocupaciones de la Edad del Bronce.

Los resultados preliminares revelan la existencia de ocupaciones de hábitat periódicas y la explotación de recursos procedentes del Valle del Ebro y de la costa atlántica, contribuyendo a reconstruir los modos de vida y la circulación de bienes cotidianos en el norte de la península ibérica.

\section{LABURPENA}

Kantauri itsasoko ertzean Brontze Aroko ohizko ikerketa luxozko ondasunen eta hileta-aztarnategien azterketara orekatu izan da. Horren ondorioz, gai historiko honi buruzko egungo egoeran hutsune larriak daude populatze eredua, eguneroko bizimoduak, eta estrategia ekonomikoak ezagutzeko.

Artikulu honetan San Adrian (Aizkorri mendilerroa, Gipuzkoa) aztarnategiko Brontze Aroko okupazioen datuak aurkezten dira, zeinen indusketari esker aztertu ahal izan ditugun aldi hartako bizimoduak, oinarri ekonomikoak, kultura materiala eta ingurumena.

Behin-behineko datuek bertan bizi-okupazioak maiz ezarri zirela eta Ebro Arroko nahiz Atlantiar itsas-ertzeko baliabideak erabili izan zirela adierazten dute, modu honetan Iberiar penintsulako iparraldean Brontze Aroko bizimoduen eta eguneroko ondasun-zirkulazioaren ezagutzari lugunduz.

\section{ABSTRACT}

Bronze Age studies carried out in the Cantabrian Region have traditionally focused on prestige goods and funerary contexts. As a result of this, the lack of information about daily activities, subsistence strategies, and human settlement on a regional scale is evident in the state of art. However, current research has achieved new discoveries in recent years, allowing a reconstruction of some aspects of the economic structure, settlements, material culture and the palaeoenvironment during the Bronze Age. Indeed, besides the funerary practices discovered in 1983 in San Adrian (Parztuergo Nagusia, Gipuzkoa), research has now revealed the presence of Upper Palaeolithic and Early Bronze Age occupations.

This paper presents a first characterization of the retrieved evidence and a preliminary evaluation of the archaeological site and its environment. San Adrian is a tunnel-shaped cave located at 1,000 meters a.s.l. in the Aizkorri mountain range, opening a passage beneath the Atlantic-Mediterranean watershed in northern Iberia. The strategic character of this mountain site is demonstrated by the presence of Upper \footnotetext{
(2) Bio ArCh, University of York.

(3) Instituto Internacional de Investigaciones Prehistóricas de Cantabria (IIIPC)-Universidad de Cantabria.

(4) 3DTS

(5) Universidad de Salamanca

(6) Ikerbasque-Basque Foundation for Science/Universidad del País Vasco-Euskal Herriko Unibertsitatea.

(7) Universidad del País Vasco/Euskal Herriko Unibertsitatea.
}

(1) Sociedad de Ciencias Aranzadi. Corresponding author: Jesús Tapia. Email: jtapia@aranzadi.eus. Zorroagagaina kalea 11, E-20014 Donostia (SPAIN). 
Palaeolithic and Bronze Age occupations, and by the construction of a road passing through it and the fortification of both its entrances in the Middle Ages.

The aim of the archaeological survey started in 2008 was to identify, describe and evaluate the heritage potential of the cave, because previous fieldwork had only managed to make surface finds in the side galleries, including a medieval hoard and Bronze Age human remains.

The work carried out by our research group at San Adrian includes a series of test pits and the excavation of an area nine square metres in size following stratigraphic criteria. In the current state, we identified at least two contexts corresponding to Late Upper Palaeolithic and Bronze Age occupations in the cave. Fieldwork included the sieving and flotation of sediment and the collection of samples for different types of analysis: palynology, carpology, sedimentology, and radiocarbon dating. The evidence is being studied by a multidisciplinary team according to expertise requirements for each topic: palaeobotany and environment, archaeozoology, sedimentology, geology, physical anthropology, prehistoric industries (lithics, pottery and bone) and archaeological and historical documentation.

Because of its recent discovery, Upper Palaeolithic evidence remains still under study, but first results on Bronze Age layers can be presented. The ongoing archaeobotanical and archaeozoological studies reveal the exploitation of domestic plants and fauna complemented by hunting and foraging of wild species. At the same time, the archaeological artefacts and their production sequences show the exploitation of nearby resources on both sides of the mountain range, while prestige goods are absent. This evidence is also used to estimate the regularity of cave occupations and to propose a model of seasonal exploitation of the mountain environment.

The results obtained reveal the exploitation of resources from both the Mediterranean and Atlantic basins, and contribute towards an understanding of the daily activities of Bronze Age societies. In addition, the evidence shows the exchange and circulation of quotidian products between the Cantabrian region and inland Iberia in other networks than those of prestige goods.

\section{1.- INTRODUCCIÓN}

Los estudios sobre Prehistoria reciente en la región cantábrica han constituido tradicionalmente un ámbito de investigación secundario en comparación con otros períodos de la Prehistoria, especialmente el Paleolítico Superior. Este sesgo es resultado de un notable desequilibrio en la cantidad y calidad de los contextos disponibles para cada ámbito cronológico, pero también se debe a una escasa renovación de los enfoques aplicados al estudio de los períodos más recientes.

A este respecto, la investigación tradicional se ha resignado a asumir la pobreza de la evidencia disponible para la Edad del Bronce y a proponer esquemas interpretativos basados en la marginalidad del área cantábrica respecto a las influencias culturales europea y peninsular.

Las evidencias conocidas para este período en la región proceden de hallazgos metálicos descontextualizados (ARMENDARIZ, 1984), de ajuares de estructuras megalíticas (MUJIKA \& EDESO, 2011), y sobre todo de yacimientos en cueva (ARMENDARIZ, 1990; ARMENDARIZ \& ETXEBERRIA, 1983), entre los que predominan los de carácter sepulcral, aunque en algunos casos se han podido identificar actividades de subsistencia (STRAUS \& GONZALEZ MORALES, 2012).

El estudio de objetos diagnósticos, sobre todo metálicos y cerámicos, ha permitido una correlación a escala regional y europea que contrasta con nuestro desconocimiento casi absoluto de los modos de vida y de organización social de los grupos humanos locales. En consecuencia, el predominio de contextos en cueva o megalíticos ha favorecido que perdure una caracterización de estas sociedades como grupos eminentemente ganaderos, con cierta jerarquía social, y subsidiarios de comunidades agrícolas vecinas como el valle del Ebro o la cuenca aquitana (APELLÁNIZ, 1973; ALDAY, 1992).

En los últimos años la excavación del yacimiento de Haltzerreka (Gipuzkoa) ha permitido documentar por primera vez en el cantábrico un lugar de hábitat del
Bronce Antiguo al aire libre, en el que se ha recuperado un variado conjunto de restos cerámicos, líticos y de macrorrestos vegetales (MUJIKA ET AL., 2009; RUIZ ALONSO, 2009).

Las excavaciones realizadas recientemente en la cueva de San Adrian (Gipuzkoa), cuyos resultados se presentan en este artículo, contribuyen a paliar este vacío en la investigación al registrar una importante secuencia de ocupaciones del Bronce Antiguo. Las evidencias documentadas en este nuevo yacimiento incluyen estructuras en fosa, macrorrestos vegetales, artefactos líticos, óseos y cerámicos, restos humanos y faunísticos, y se han podido obtener dataciones radiocarbónicas y datos paleobotánicos y sedimentológicos. Nuestra interpretación y contextualización de estos datos permite perfilar un modelo de subsistencia agropecuario, y una explotación de recursos cotidianos del entorno que sirve de contrapunto a la circulación de objetos de prestigio a escala peninsular y europea.

\section{2.- LOCALIZACIÓN Y DESCRIPCIÓN DEL YACI- MIENTO}

San Adrian es una cueva en forma de túnel situada a 1.035 m.s.n.m. y abierta en dirección SW-NE en la Sierra de Aizkorri (Gipuzkoa), formando parte de los Montes Vascos que enlazan los Pirineos con la Cordillera Cantábrica en el norte de la Península Ibérica. La cueva atraviesa un farallón de calizas cretácicas (Aptiense superior) en la divisoria de aguas atlántico-mediterránea, y constituye un paso natural entre el Golfo de Vizcaya y el interior peninsular, comunicando la costa cantábrica y el Valle del Ebro a través de la sierra.

El tramo principal de la cueva está constituido por un conducto de 60 metros de longitud, con una anchura variable de entre 10 y 20 metros, y una altura máxima de 20 metros sobre el nivel de relleno actual. En el lado noreste se abre una entrada de más de 15 metros de ancho y 20 metros de altura, mientras que la boca suroeste presenta unas dimensiones menores, de aproxi- 
madamente 6 metros de ancho por 2,5 metros de alto. Este conducto principal se formó por procesos freáticos controlados por una falla de dirección W-E, mientras que en el lado sur se desarrolló una red de galerías secundarias que quedaron colgadas sobre el tramo principal de la cueva (FIGURA 1).
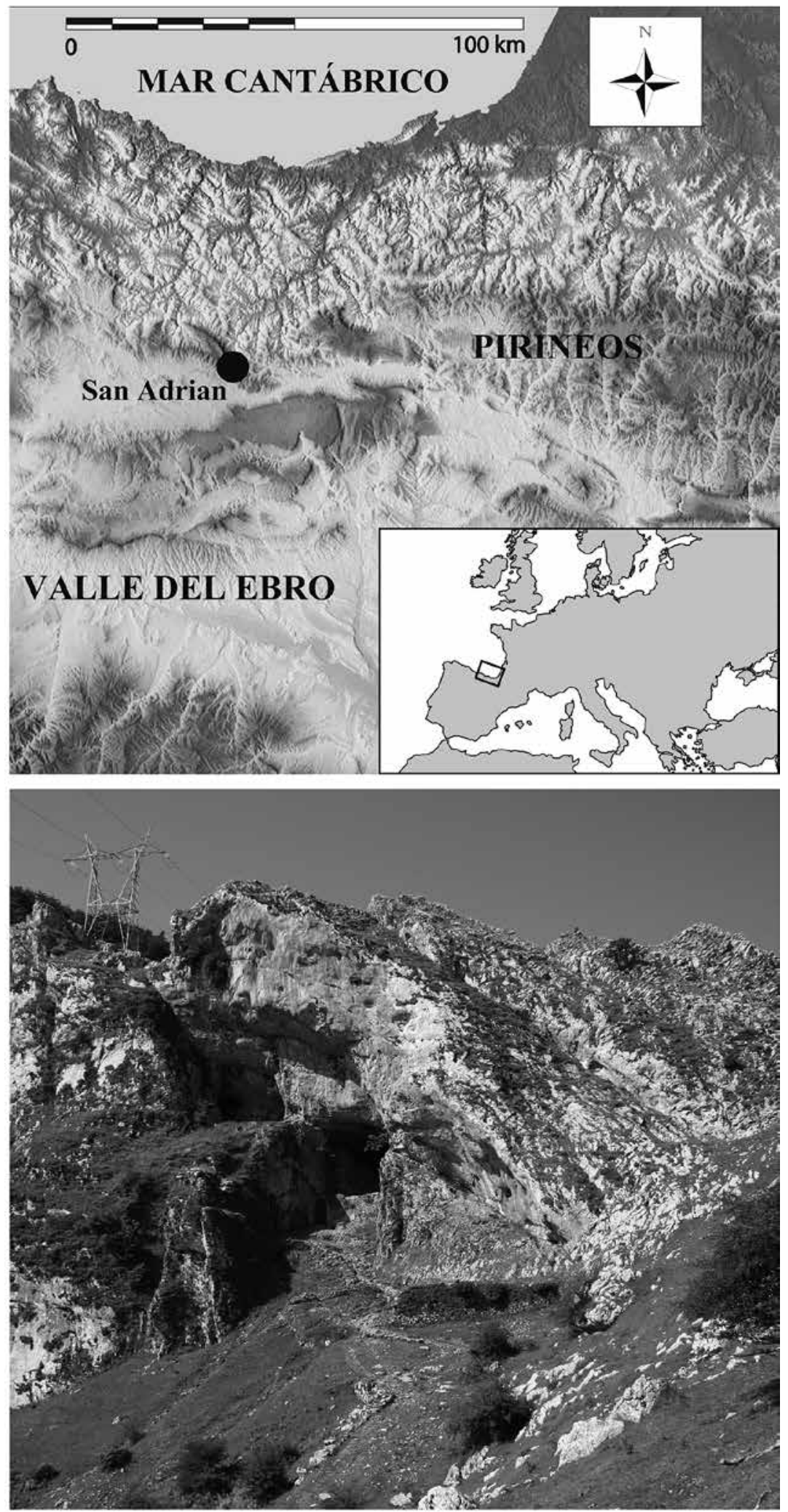

Fig. 1. Localización y vista general de San Adrian / Location and general view of San Adrian cave. 
JESÚS TAPIA, MIRIAM CUBAS, MANUEL CEBERIO, ALFREDO MORAZA, JUANTXO AGIRRE-MAULEON, EUKEN ALONSO, ESTEBAN ÁLVAREZ-FERNÁNDEZ, PABLO ARESO, ÁNGEL ARMENDARIZ, PEDRO CASTAÑOS, JONE CASTAÑOS, FRANCISCO ETXEBERRIA, JOSEBA GARMENDIA, LOURDES HERRASTI, MARÍA JOSÉ IRIARTE CHIAPUSSO, DANIEL PÉREZ, ANA URIZ, LYDIA ZAPATA

La importancia del paso de San Adrian se ve corroborada por la existencia de distintas estructuras defensivas en el entorno de la cueva desde al menos la Edad Media (s. X-XVI). Además de ellas se puede documentar otra serie de construcciones relacionadas fundamentalmente con el carácter estratégico de este emplazamiento (distintas calzadas, un hostal, almacenes, y cuadras, ermita, una casa de guardia, etc.) y cuyos últimos habitantes abandonaron el lugar a principios del s. XX.

A pesar de su espectacularidad, la cueva-fortaleza de San Adrian no ha sido objeto de intervenciones arqueológicas sistemáticas, y los estudios históricos hasta el presente se han centrado en las fuentes documentales y en los hallazgos metálicos medievales recuperados en una galería lateral (GARCÍA RETES, 1987).

En el entorno inmediato se conocía la existencia de otros yacimientos prehistóricos, como la cueva sepulcral de Partxan Kobia, el túmulo de San Adrian, o el hallazgo del hacha pulimentada de Aratz (ALTUNA ET AL., 1982), y una exploración realizada en 1984 permitió recuperar en las galerías laterales de San Adrian restos humanos de al menos dos individuos (ALTUNA ET AL., 1995). Sin embargo, la existencia de estructuras construidas ha impedido valorar correctamente el potencial estratigráfico del tramo central de la cueva.

\section{3.- INTERVENCIÓN ARQUEOLÓGICA: 2008- PRESENTE}

\subsection{Excavación}

Desde 2008 desarrollamos un proyecto de investigación sobre San Adrian que incluye el análisis de la documentación histórica, la realización de excavaciones arqueológicas y sondeos en la cueva y en su entorno inmediato, y un programa de restauración de las estructuras conservadas.

La excavación de un sondeo en lado $\mathrm{W}$ de la ermita en 2008 permitió identificar una importante secuencia estratigráfica en el tramo central del túnel, localizándose un nivel de ocupación con restos de fauna y cerámicas de la Edad del Bronce. Posteriormente, la retirada de un potente nivel de escombros en el lado opuesto de la ermita puso al descubierto un nuevo tramo de la secuencia estratigráfica, que está siendo excavado en una extensión de 9 metros cuadrados desde 2011 (FIGURA 2).

Esta excavación de los niveles de la Edad del Bronce se realizó manualmente, atendiendo a criterios estratigráficos y se registraron los distintos depósitos y estructuras siguiendo el método Harris (HARRIS, 1991). Todo el sedimento fue revisado mediante cribado (2mm) y los materiales recuperados fueron registrados con referencia a su unidad estratigráfica de procedencia.

Durante la excavación se reconocieron diversos depósitos y estructuras negativas, cuyos contenidos y rellenos fueron excavados y procesados de forma individualizada, y se tomaron distintas muestras para dataciones de C14 y análisis sedimentológicos y paleobotánicos (flotación y muestreos palinológicos).

En 2012 realizamos un sondeo de 1,5x1 metros para evaluar el potencial arqueológico del depósito previo a la Edad del Bronce, detectando una nueva secuencia de ocupaciones del Magdaleniense Superior, cuya excavación estamos ampliando actualmente a toda el área.

\subsection{Estratigrafía}

La secuencia de ocupaciones que presentamos en este artículo corresponde a los niveles adscritos a la Edad del Bronce y a los inmediatamente infra- y superpuestos dentro del área de excavada en 2011. Como se ha explicado anteriormente, el descubrimiento de este depósito se produjo tras la retirada de un potente nivel de rellenos de escombro situado al lado $E$ de la ermita. Cubierto por este nivel se identificó un habitáculo cuadrangular construido mediante tres muros de mampostería (UUEE 1206, 1207 y 1208) adosados a la pared $\mathrm{N}$ del conducto, que delimitan el área excavada desde 2011. Bajo el nivel de escombros que rellena y cubre este espacio se identificó un primer nivel de ocupación a base de tierras apelmazadas (UE 1201) que contenía materiales bajomedievales, de época moderna, y de la Edad del Bronce, así como otros depósitos de menor extensión y de cronología igualmente post-medieval (UE 1203).

Bajo este primer nivel de acondicionamiento del piso de la estancia se desarrolla el resto de la secuencia sin que hayamos detectado nuevas intrusiones de materiales modernos.

La secuencia de la Edad del Bronce se inicia con la UE 1202, un depósito de color negruzco debido a la presencia de restos vegetales carbonizados y con abundantes restos arqueológicos, principalmente cerámicos y faunísticos. A medida que excavamos este primer depósito fueron apareciendo diversas cubetas y depresiones excavadas en el suelo. Todas las estructuras aparecen cubiertas por la UE 1202, y al mismo tiempo algunas de ellas están excavadas en la parte inferior de la misma, de modo que decidimos dividir la UE 1202 en dos tramos (superior e inferior) a pesar de que, atendiendo a criterios sedimentológicos, no se apreciara solución de continuidad entre ambos (FIGURA 3).

Todas las fosas salvo la UE 1221 son de delineación circular y de pequeña extensión (1209, 1213, $1215,1216,1250,1251$ y 1252), afectando de forma variable al resto de depósitos infrapuestos, pero sin llegar a traspasar en general la base de la secuencia de ocupaciones del Bronce (UE 1204). Algunas de ellas pueden interpretarse como silos o pequeños depósitos, mientras que los de perfil más abierto y de menor diámetro y profundidad pueden considerarse cubetas de hogar o similares. En algunos casos se han podido distinguir depósitos de relleno independientes para cada estructura negativa, mientras que en otros casos su relleno es indiferenciable de la UE 1202. 

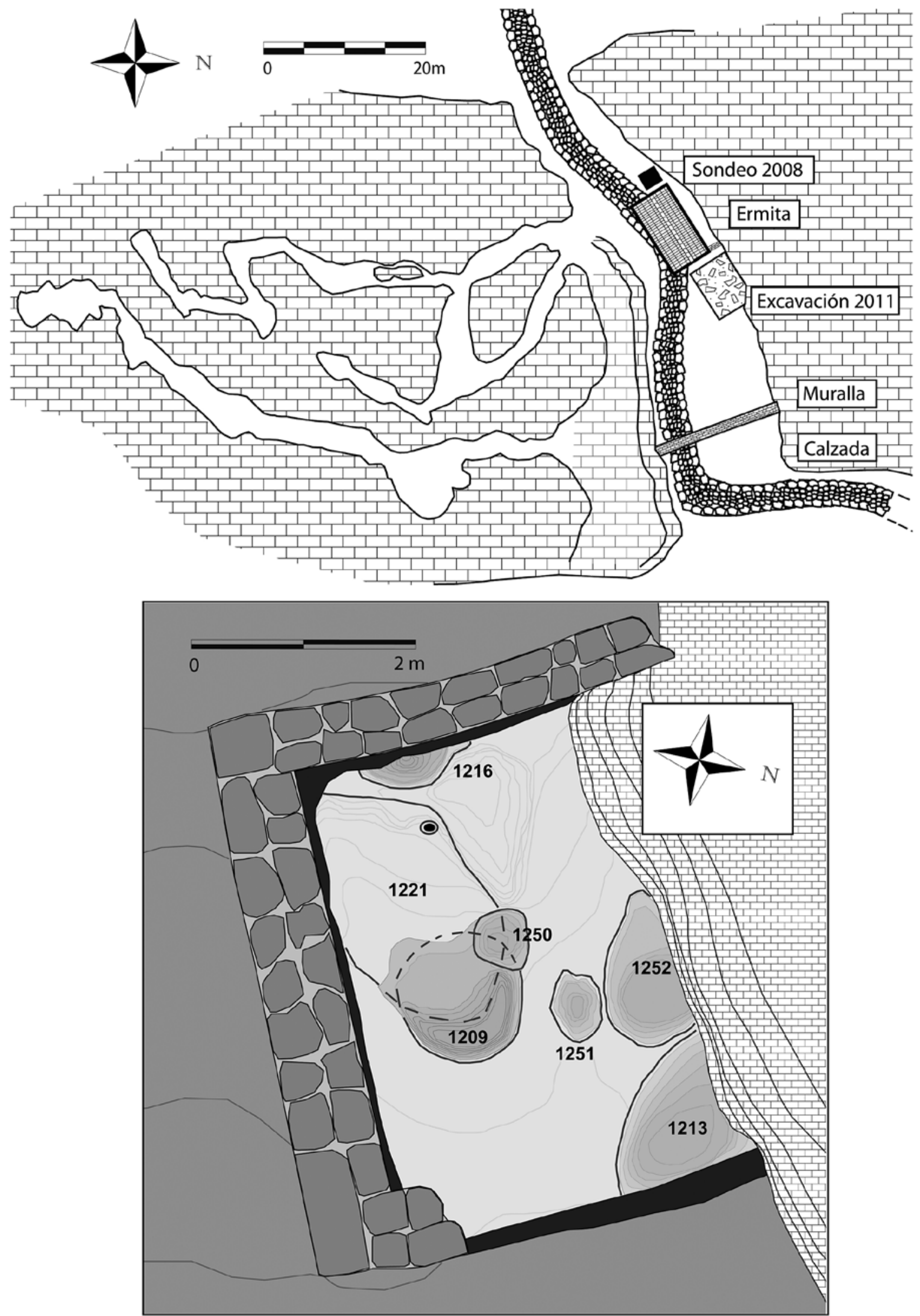

Fig. 2. Plano general de San Adrian y detalle de la ocupación de la Edad del Bronce / General plan of San Adrian, and detail of the Bronze Age occupation. 


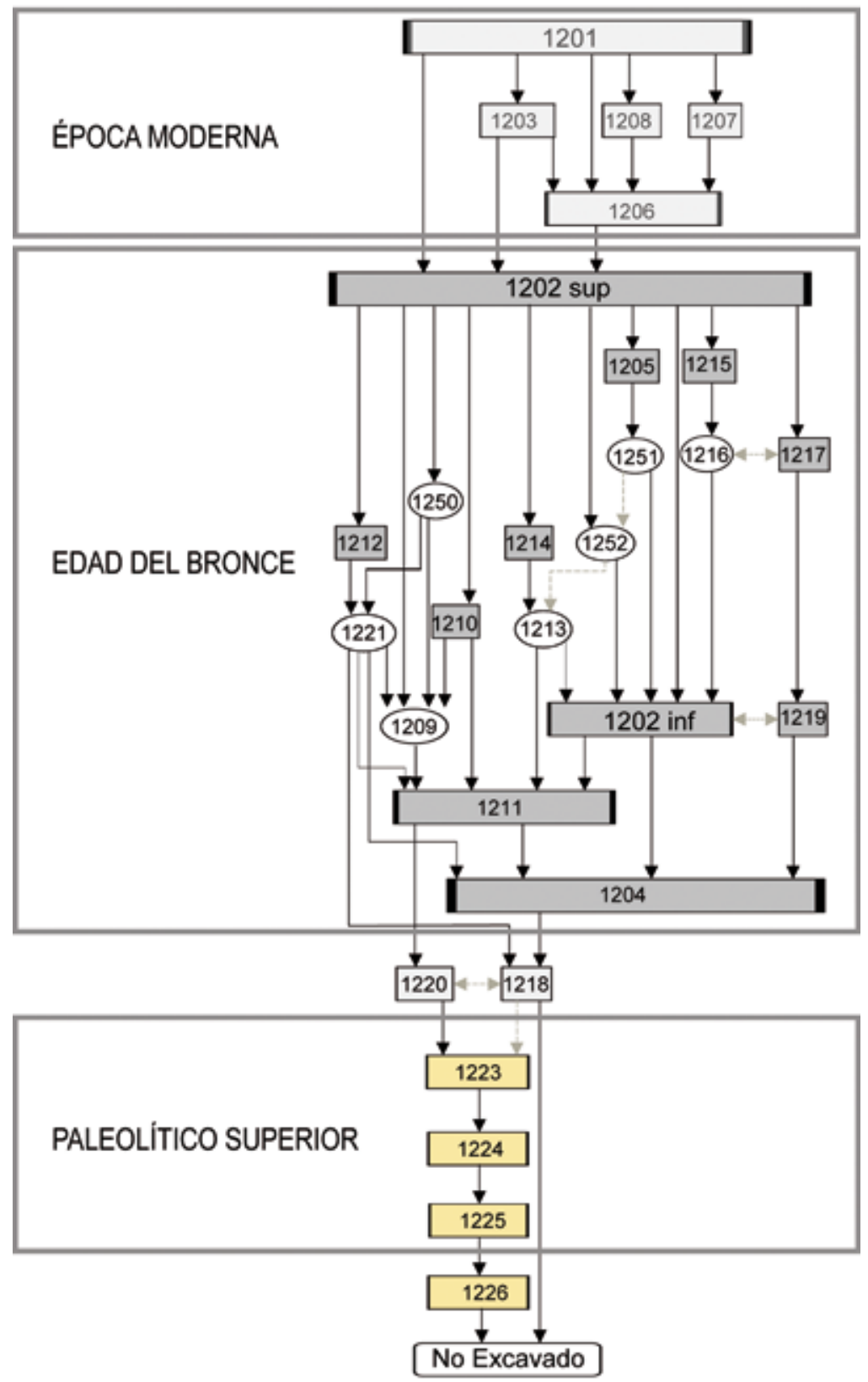

Fig. 3. Diagrama de la secuencia estratigráfica de San Adrian (2011). Modificado a partir de Harris matrix composer 2.0a (Copyright by Imagination Computer Services 2013) / Diagram of the stratigraphic sequence of San Adrian (2011). Adapted from Harris matrix composer 2.0a (Copyright by Imagination Computer Services 2013)

La excepción dentro de estas estructuras la constituye la UE 1221, que interpretamos como un posible fondo de cabaña. Se trata de una depresión de tamaño considerablemente mayor y con planta de tendencia oval, y en cuya base se identificó un pequeño orificio relleno de materia carbonosa -¿hoyo de poste?-. Sin embargo la estructura no ha podido ser analizada en su totalidad ya que se prolonga más allá del área de excavación delimitada por los muros modernos.

Debe destacarse el hecho de que varias de estas fosas se encuentran recortadas o interrumpidas entre sí. Al menos 3 de ellas (1250, 1221 y 1209 de más reciente a más antigua) se encuentran claramente superpuestas y en contacto físico, y para otras $3(1213,1252$ y 1251) puede suponerse un orden de antecedencia atendiendo a criterios de operatividad y de disponibilidad de espacio.

Todos los niveles de esta secuencia estratigráfica contienen materiales arqueológicos asignables al Bronce Antiguo, sin posibilidad de establecer mayores distinciones, hasta la UE 1204, por debajo de la cual -en términos estratigráficos- no se han localizado materiales arqueológicos atribuibles a este período.

Bajo esta secuencia de la Edad del Bronce se han identificado otros depósitos arqueológicamente estériles, y a continuación una nueva secuencia de ocupaciones con abundante material (fauna e industria lítica) 
correspondiente a sucesivas ocupaciones de finales del Paleolítico Superior (1223, 1224 y 1225).

A pesar de que en la unidad estratigráfica más antigua (1226) no se han localizado de momento restos arqueológicos, para el relleno sedimentario de la cueva -cortado por la muralla medieval en la boca E- puede estimarse una potencia de al menos otros 5 metros de profundidad.

\subsection{Sedimentología}

La identificación en campo de las unidades estratigráficas se ha basado en la distinción de rasgos macroscópicos (texturas, contenido de clastos y tonalidad del sedimento) dentro de la homogeneidad que presenta el relleno de la cueva.

El análisis sedimentológico, realizado en laboratorio a partir de muestras de distintas unidades estratigráficas, confirma esta homogeneidad y permite incluirlas en un mismo proceso de sedimentación con leves oscilaciones.

Los materiales del relleno provienen de la disgregación y disolución in situ de las calizas Urgonianas y de algunos aportes de sedimento introducidos por la corriente aérea que circula por el túnel. Los rasgos que caracterizan el depósito son porcentajes elevados de cantos calizos, muy poca concreción, elevado contenido de iones carbonatados en el sedimento, y una escasa representación de materiales alóctonos.

La altitud y la orientación de túnel lo sitúan en una zona estacionalmente muy fría, donde los cantos y gránulos, casi siempre calizos, superan en todas las muestras el $60 \%$ del sedimento, y con frecuencia tienen forma de crioclastos bastante alterados.

La fracción fina no supera nunca el 40 \% del sedimento, y no hay diferencias apreciables entre los rellenos de cubetas y la columna, predominando siempre los calibres más finos (arcillas, limos y arenas finas). La morfoscopía de las arenas encuentra pocos cristales cuarcíticos, que aparecen algo rodados y con superficies mate, indicando un posible origen eólico.

El contenido en carbonatos es particularmente alto (entre el 42 y $52 \%$ ) y se ha producido un lavado de iones CO3- en los niveles superiores y una ligera acumulación en horizontes inferiores, debido a las condiciones de humedad ambiental y a la porosidad del relleno sedimentario. Las diferentes muestras estudiadas son muy homogéneas entre sí en lo que se refiere al reparto de materiales gruesos y de la matriz. Las pequeñas diferencias apreciadas en alguno de los parámetros medidos no se asocian a cambios significativos en el proceso de sedimentación, sino a oscilaciones en dicho proceso.

\subsection{Cronología del depósito}

Las dos dataciones por C14 obtenidas de sendos tramos del depósito confirman la atribución cronológica de la secuencia excavada a la Edad del Bronce, mientras que otras dos permiten correlacionarla con el nivel de ocupación detectado en el lado opuesto de la ermita en 2008, y con los restos humanos recogidos en las galerías en 1984.

Estas dataciones se agrupan en el intervalo 18801510 cal BC de manera solapada, y sólo la correspondiente a la UE 1202-superior aporta una fecha algo más antigua. Consideramos que esta inversión respecto a la datación de la UE 1214 (relleno de la cubeta 1213) no es especialmente relevante, ya que puede deberse a diversas alteraciones del depósito provocadas durante las ocupaciones (TABLA I).

Por otra parte, resultados preliminares sitúan las ocupaciones detectadas bajo la secuencia del Bronce a finales del paleolítico Superior (12.000-11.000 BP).

\section{4.- RESTOS ARQUEOLÓGICOS}

\subsection{Fauna}

A partir de la excavación se ha recuperado un total de 1823 fragmentos de hueso de los cuales han sido susceptibles de identificación 786, que representan algo menos de la mitad $(42,5 \%)$ de todo lo recogido. Esta acumulación de restos proporciona una información valiosa acerca de las bases de subsistencia de origen animal del grupo humano. El grado de fragmentación que presenta la muestra es habitual en yacimientos arqueológicos y confirma que la mayor parte de la fauna procede de animales sacrificados y consumidos por los ocupantes del asentamiento.

Los datos obtenidos muestran un claro predominio de las especies domésticas frente a la pequeña explotación de los recursos silvestres. Entre las especies domésticas predomina el ganado vacuno $(56,8 \%)$, rasgo

\begin{tabular}{|c|c|c|c|c|c|c|}
\hline UE & MUESTRA & MÉTODO & REF. LAB. & DATACIÓN BP & CAL BP $(2 \sigma)$ & CAL BC $(2 \sigma)$ \\
\hline Sondeo 2008 & Hueso & C14 AMS & Ua-37522 & $3430 \pm 40$ & $3830-3590$ & $1880-1640$ \\
\hline Superficial (1984) & H. humano & C14 AMS & Ua-44255 & $3311 \pm 30$ & $3610-3460$ & $1660-1510$ \\
\hline UE 1214 & Bellota & C14 AMS & Ua-44254 & $3378 \pm 30$ & $3700-3560$ & $1750-1610$ \\
\hline UE 1202-sup & Hueso & C14 AMS & Ua-44256 & $3599 \pm 32$ & $3980-3830$ & $2040-1880$ \\
\hline
\end{tabular}

Tabla 1: Dataciones de las secuencias de San Adrian. La calibración de las dataciones se ha realizado con la curva IntCal13 (REIMER, P.J. ET AL., 2013) para muestras de origen terrestre y con el programa OxCal 4.24 (RAMSEY, C. B., 2001, 2009). Las dataciones se expresan con un intervalo de probabilidad 20, en años "cal BC" y "cal BP" / Radiocarbon dates for the stratigraphic sequence of San Adrian, calibrated with the IntCal13 curve (REIMER, P.J. ET AL., 2013) for terrestrial samples using the OxCal program (RAMSEY, C. B., 2001, 2009). Results are shown in "cal BC" and "cal BP" years with a confidence interval of 20. 
JESÚS TAPIA, MIRIAM CUBAS, MANUEL CEBERIO, ALFREDO MORAZA, JUANTXO AGIRRE-MAULEON, EUKEN ALONSO, ESTEBAN ÁLVAREZ-FERNÁNDEZ, PABLO ARESO, ÁNGEL ARMENDARIZ, PEDRO CASTAÑOS, JONE CASTAÑOS, FRANCISCO ETXEBERRIA, JOSEBA GARMENDIA, LOURDES HERRASTI, MARÍA JOSÉ IRIARTE CHIAPUSSO, DANIEL PÉREZ, ANA URIZ, LYDIA ZAPATA

típico de los yacimientos de la Edad del Bronce de la cornisa cantábrica y de la meseta Norte. Siguen en importancia el ganado ovicaprino $(32,1 \%)$ con presencia de restos tanto de oveja como de cabra. La representación de estas dos últimas junto con la presencia de perro indica la existencia de un componente pastoril en la economía pecuaria del asentamiento. El ganado porcino aunque en menor proporción completa el cuadro de subsistencia animal del grupo que ocupó el yacimiento (TABLA II).

\begin{tabular}{|l|c|c|c|}
\hline \multicolumn{1}{|c|}{ Especies } & $\begin{array}{c}\text { UE } \\
\mathbf{1 2 0 2}\end{array}$ & $\begin{array}{c}\text { Otras } \\
\text { UE }\end{array}$ & Total \\
\hline Vacuno (Bos taurus) & 438 & 21 & 459 \\
\hline Ovicaprino (Ovis aries) & 213 & 19 & 232 \\
\hline Cerdo (Sus domesticus) & 76 & 5 & 81 \\
\hline Perro (Canis familiaris) & 3 & 1 & 4 \\
\hline Cabra montesa (Capra pyrenaica) & 6 & & 6 \\
\hline Ciervo (Cervus elaphus) & 1 & & 1 \\
\hline Corzo (Capreolus capreolus) & 2 & & 2 \\
\hline Buitre leonado (Gyps fulvus) & 1 & & 1 \\
\hline TOTALES & $\mathbf{7 4 0}$ & $\mathbf{4 6}$ & $\mathbf{7 8 6}$ \\
\hline
\end{tabular}

Tabla 2: Restos de fauna (NR) recuperados en la secuencia de la Edad de Bronce de San Adrian / Faunal remains (NR) retrieved from the Bronze Age sequence of San Adrian.

El modelo de aprovechamiento pecuario es de tipo mixto tanto en el ganado vacuno como en el ovicaprino, y en el cerdo es de carácter predominantemente primario. El sacrificio de los individuos en las cabañas pastoriles está repartido entre las distintas cohortes de edad, indicando que unos animales (infantiles y juveniles) se mataron con el fin de aprovechar fundamentalmente su carne y otros (adultos) se conservaron de cara a la obtención de productos secundarios -en el caso del vacuno para leche y trabajo y en el ovino para lana-. La matanza de cerdo se circunscribe al periodo entre uno y dos años, lo que indica que esta cabaña se destina sobre todo a la obtención de productos cárnicos y el sacrificio coincide con el momento de mejor relación edad-peso.

La representación de huesos del tronco de las distintas especies revela también, para el ganado vacuno y ovicaprino, un patrón distinto al del porcino. En el primer caso los huesos del tronco (vértebras y costillas) representan una tercera parte de todos los restos, lo que sugiere que las reses fueron sacrificadas en el mismo yacimiento. En cambio, en el caso del cerdo los elementos del esqueleto axial sólo representan la décima parte del total, escasez que puede atribuirse también a diferencias de conservación y consumo entre algunas partes del cuerpo, como los costillares, con relación a otras como los brazuelos y los jamones.

Finalmente, la escasa proporción de huesos pertenecientes a especies salvajes indica que esta forma de explotación del territorio fue muy secundaria, reduciéndose a la caza de cabra montés, corzo y quizás ciervo, ya que hay una espátula fabricada con asta de esta última especie aunque podría proceder de la recolección de alguna cornamenta de desmogue. Entre las especies salvajes destaca por su rareza la presencia del buitre leonado.

\subsection{Restos paleobotánicos}

Los resultados preliminares de los análisis paleobotánicos permiten reconstruir un paisaje abierto, con una reducida cubierta arbórea como consecuencia de la acción humana. Las ocupaciones humanas ejercieron una importante presión antrópica sobre el entorno de San Adrian, lo que supuso el predominio de los espacios abiertos y de las especies herbáceas-arbustivas frente al bosque, formado por una diversidad de especies arbóreas caducifolias (avellanos, abedules, robles, tilos, fresnos y hayas) que conviven con el pino.

En el estrato herbáceo dominado por las gramíneas están representados también brezos, leguminosas, ranunculáceas y liliáceas, entre otros taxones. El grado de humedad era importante como lo indica la representación de esporas de helechos y de especies de plantas asociadas a ambientes húmedos como las ciperáceas, y destaca el aliso dentro de la vegetación de ribera.

Los macrorrestos vegetales recuperados mediante la flotación del sedimento se han conservado carbonizados y presentan un alto grado de fragmentación $(1 \mathrm{~cm}$ o menor). Entre los fragmentos de madera carbonizada existe una interesante diversidad de especies, en los que destacan avellano, tejo, abedul, fresno y roble, que pueden asociarse con su consumo como combustible, pero sin descartarse su empleo en estructuras constructivas o en la elaboración de útiles y objetos.

Un aspecto relevante, puesto de manifiesto en este estudio, es la confirmación de la existencia de campos de cultivo en el entorno cercano al yacimiento, como lo certifican los pólenes de Cerealia y las semillas recuperadas. Entre las semillas identificadas hasta el momento, la mayor parte corresponden a semillas de trigo común así como a algunas malas hierbas, habitualmente asociadas a los campos de cultivo. También se han recuperado restos de avellanas y bellotas, alimentos que tienen una larga tradición de uso entre los grupos humanos prehistóricos europeos.

\subsection{Restos cerámicos}

La cerámica constituye el material arqueológico más abundante entre los procedentes de la ocupación de la Edad del Bronce. Sin embargo, el conjunto presenta un elevado índice de fragmentación, por lo que no ha podido documentarse la presencia de recipientes completos, aunque el remontaje de diversos fragmentos ha permitido la reconstrucción de algunas morfologías. 
El mero análisis macroscópico realizado hasta el momento no permite identificar de manera precisa las distintas materias primas empleadas en su elaboración, aunque sí ha posibilitado la identificación de distintos tipos de inclusiones no plásticas, tanto de naturaleza mineral -calcita-, como artificial -chamota (presente en varios de los fragmentos examinados)-.

El conjunto cerámico $(\mathrm{NR}=1599)$ presenta una manufactura a mano. En general, no se observan trazas tecnológicas que permitan inferir la técnica de elaboración, aunque algunos fragmentos permiten intuir una manufactura a rollos. La muestra conservada refleja la existencia de recipientes cerámicos de cierta diversidad que a grandes rasgos pueden clasificarse en tres categorías: grandes vasos -generalmente decorados mediante cordones con digitaciones-, vasos de tamaño mediano o pequeño -generalmente lisos-, y otros, también de pequeño o mediano tamaño, más cuidados y con decoraciones menos comunes. En todos los casos, las superficies aparecen generalmente regularizadas, aunque también se observan otros tratamientos superficiales como el alisado, el bruñido, el raspado o la aplicación de barro plástico. Estos tratamientos se encuentran tanto en las superficies internas como externas, exceptuando la aplicación de barro plástico, que aparece exclusivamente en el exterior de los recipientes.

Para su análisis, los fragmentos se han clasificado como: borde, cuello, galbo, galbo indeterminado, elemento de prensión y base. Los primeros presentan distintas direccionalidades (recta, exvasada o vuelta al interior) y diferentes morfologías (redondeada, engrosada, plana,...). Los cuellos son mayoritariamente cóncavos mientras que los galbos presentan una mayor variabilidad formal, identificándose galbos convexos, rectos o carenados. Entre los elementos de prensión destaca la presencia de mamelones de distintas morfologías, lengüetas y asas de cinta. La parte morfológica que refleja una menor variabilidad formal es la base, plana en todos los casos, aunque en ocasiones aparece ligeramente indicada.

Como ya se ha dicho, durante la intervención arqueológica no fue posible documentar recipientes cerámicos completos, aunque las posteriores labores de remontaje han permitido reconstruir ciertas morfologías, correspondientes a algunas de las vasijas de mayor tamaño (UE 1202). Aun incompletas, proporcionan una idea bastante aproximada de su aspecto general (FIGURA 4). Son las siguientes:

- Recipiente 1.- 35 fragmentos permiten reconstruir la mitad superior de un vaso de perfil carenado, con el borde redondeado y ligeramente exvasado. Posee un diámetro de $22 \mathrm{~cm}$ en la boca y aproximadamente 23,5 centímetros en la carena. Presenta un cordón paralelo al borde del que arrancan otros perpendiculares que se interrumpen en la zona de la carena; en todos los casos, los cordones aparecen combinados con digitaciones. Dada la escasa variabilidad entre las bases recuperadas podemos inferir una superficie de apoyo plana.

- Recipiente 2.- Parcialmente reconstruido a partir de 20 fragmentos, el diámetro aproximado de su boca es de $27 \mathrm{~cm}$, a partir de un fragmento de borde de direccionalidad recta y morfología redondeada. La mayor parte del perfil reconstruido corresponde a la parte central del recipiente, de morfología ovoide, de la que tampoco se puede identificar su base, que cabe suponer igualmente plana. Su decoración se compone de un cordón con digitaciones en el tercio superior del recipiente y un recubrimiento de barro plástico con huellas de dedadas en la parte central e inferior.

- Recipiente 3.- Reconstruido muy parcialmente en su parte central a partir de 24 fragmentos. Se trata de una vasija semejante a la anterior, de morfología ovoide con aplicación de barro plástico en la superficie exterior y un cordón con digitaciones.

La mayor parte del conjunto cerámico no presenta decoración aunque el porcentaje de fragmentos decorados es relativamente elevado (32,89\%), si se incluyen los fragmentos con aplicación de barro plástico en la superficie exterior, que constituyen un el 56,27 \% del total y un $14,38 \%$ de los decorados.

Las técnicas decorativas representadas son la impresión, la incisión y los cordones aplicados o resaltados. Estos últimos, generalmente situados cerca de la boca, constituyen el patrón decorativo más común, en combinación con la impresión de digitaciones y/o ungulaciones sobre los mismos (Figura 4.A). En general, se trata de cordones horizontales, aunque en algún caso (recipiente 1) se reconoce la presencia de cordones verticales. Dentro de la técnica impresa, a las abundantes digitaciones y ungulaciones se une la presencia, en un par de fragmentos, de pequeñas improntas circulares, realizadas mediante un instrumento hueco (Figura 4.D). Destacan igualmente algunos ejemplares (13 fragmentos) de "cerámica cordada" (Figura 4.B), en las que se imita la impronta de una cuerda posiblemente mediante la realización de pequeñas impresiones oblicuas paralelas, dispuestas horizontal o verticalmente. La técnica de decoración incisa se encuentra muy escasamente representada (sólo 2 fragmentos) (Figura 4.C), con motivos geométricos a base de líneas paralelas o en zigzag. Dentro esta técnica, puede incluirse también un tipo de decoración obtenido mediante el peinado de la superficie, del que existen algunos ejemplares.

En resumen, los rasgos morfológicos y decorativos de las cerámicas documentadas en San Adrian comparten ciertos rasgos característicos con otros conjuntos del entorno de cronología similar (Bronce Antiguo-Medio). Así ocurre, por ejemplo, con la presencia de las formas carenadas, las orzas decoradas con cordones y barro plástico, las superficies bruñidas, la tipología de los elementos de prensión y ciertos elementos decorativos, como los cordones con impresiones, motivos geométricos incisos y decoraciones cordadas. 


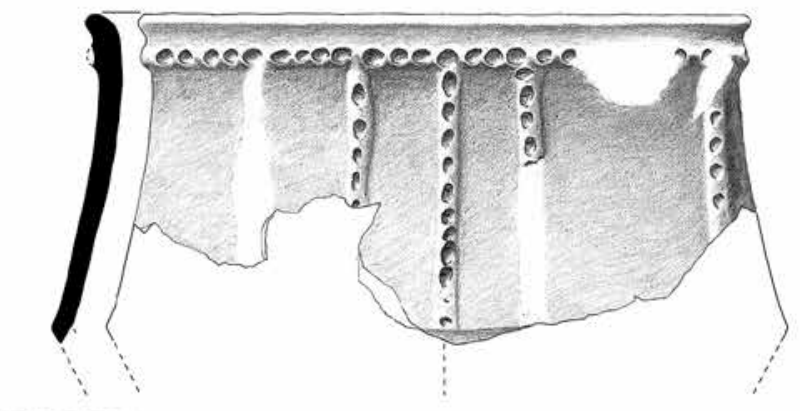

RECIPIENTE 1
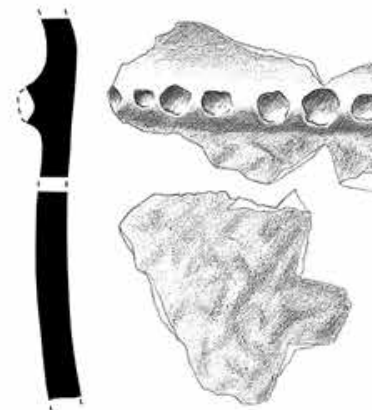

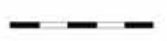

RECIPIENTE 3

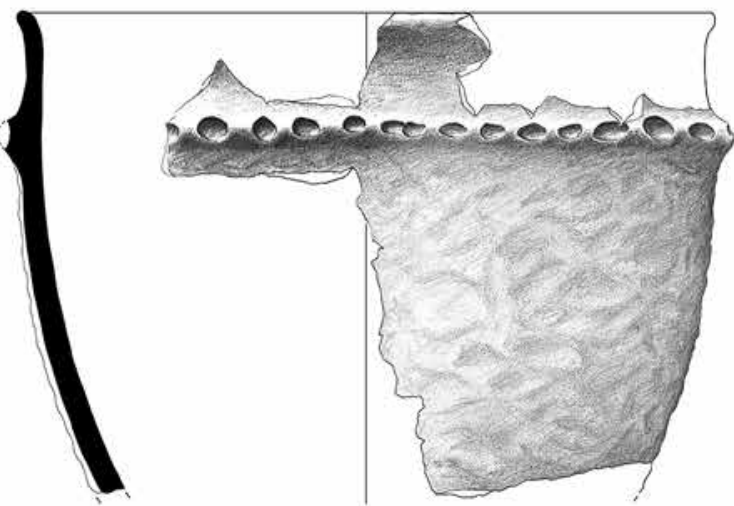

RECIPIENTE 2
A

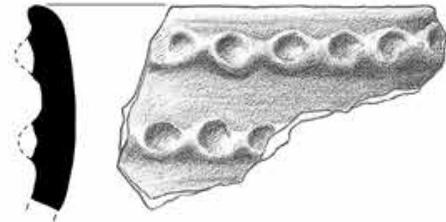

$\mathrm{C}$

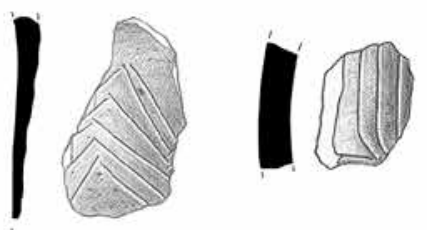

B
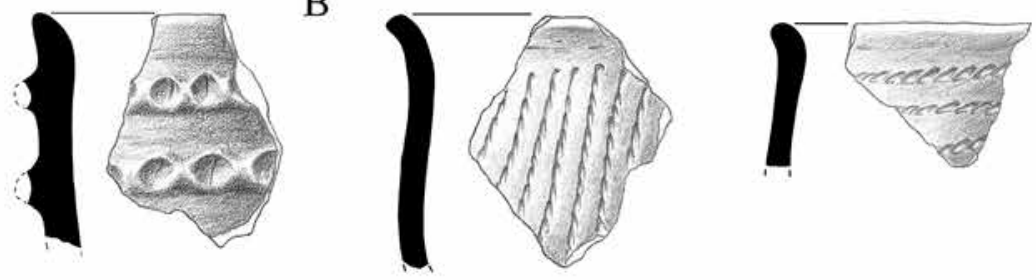

D

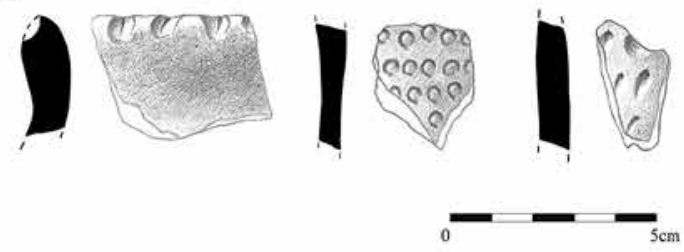

Fig. 4. Cerámica de San Adrian. Reconstrucción de los recipientes 1 a 3 y técnicas decorativas. A: cordones con ungulaciones y digitaciones; B: "cordada"; C: incisa; D: improntas circulares (Dibujos: A. Armendariz) / Pottery from San Adrian. Reconstruction of recipients 1 to 3 and decorative techniques. A: cords with nail and finger impressions; B: "corded"; C: incision; D: circular imprints (Drawings: A. Armendariz).

Estas últimas, por su técnica peculiar y poco común, adquieren especial interés, con paralelos al norte de los Pirineos, pero también dentro del País Vasco peninsular. A este respecto, destaca especialmente el asentamiento al aire libre de Haltzerreka (Idiazabal), donde se han localizado cerámicas con este tipo de decoración, acompañadas por otras que presentan motivos decorativos también semejantes a los de San Adrián, como las líneas incisas en zig-zag. Por otra parte, las mismas decoraciones cordadas se encuentran en cerámicas pro- cedentes de los cercanos sepulcros megalíticos de Napalatza (Idiazabal) y Otsaarte (Parzonería de Altzania).

\subsection{Industria lítica y ósea}

El conjunto lítico de San Adrian está formado por 227 restos en sílex, de los cuales 126 (55,5\%) presenta retoques o melladuras que sugieren su empleo como herramientas. Aunque no se ha abordado un examen exhaustivo de la procedencia del sílex, puede avanzar- 
se la posible presencia de variedades de Urbasa, del Flysch, de Treviño, y evaporítico del Ebro.

La presencia de algunos núcleos, de restos de taIla, y de un elevado número de elementos corticales, indica que algunas de estas variedades se han tallado en el mismo asentamiento. Los soportes laminares representan menos de una cuarta parte de la muestra $(22,9 \%)$, y son muy escasos los elementos de preparación y mantenimiento de los núcleos. Los formatos y siluetas de los soportes laminares son irregulares y sinuosos, con negativos unipolares, y talones predominantemente lisos. Los soportes enteros y los ejemplares sobrepasados revelan la producción de formatos cortos, de entre 2,5 y $5 \mathrm{~cm}$ de longitud.

En cuanto al abundante utillaje, el grupo más numeroso está compuesto por soportes con retoques o filos mellados, seguido a gran distancia por muescas y denticulados, raspadores, dorsos, y algunas truncaduras y buriles. Como elementos característicos destacan un elemento de hoz con lustre de cereal, un fragmento de lámina se sección trapezoidal en sílex evaporítico, y una punta de dorso con fractura de impacto en el ápice. Las dimensiones de los soportes sugieren que tanto el diente de hoz como la lámina de sección trapezoidal, obtenida mediante presión, fueron elaborados fuera del yacimiento. En cuanto a la punta, localizada en tramo inferior de la secuencia (UE 1202-inf) podría proceder de estratos inferiores removidos al cavar las cubetas (FIGURA 5).

Por tanto, se identifica una producción in situ destinada a la obtención de lascas, lascas laminares y láminas cortas, a partir de núcleos que llegan al asentamiento en forma de lascas corticales, y que se explotan siguiendo esquemas unipolares y bipolares (lascas laminares, láminas y laminillas), o centrípetos y ortogonales (lascas). Este modelo de explotación expeditiva observada en las variedades más comunes (Urbasa y Flysch) contrasta con la presencia de elementos de procedencia lejana y de mayor formato, obtenidos mediante procedimientos más cuidados, que llegan al yacimiento plenamente configurados.

En cuanto a la industria ósea, se ha recuperado una espátula en asta de ciervo y dos útiles en hueso. El primero de ellos es un fragmento de varilla cilíndrica realizada a partir de un hueso de gran herbívoro, y el segundo es un fragmento de punzón de sección triangular con un intenso pulido.

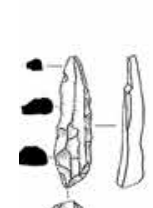

(1)
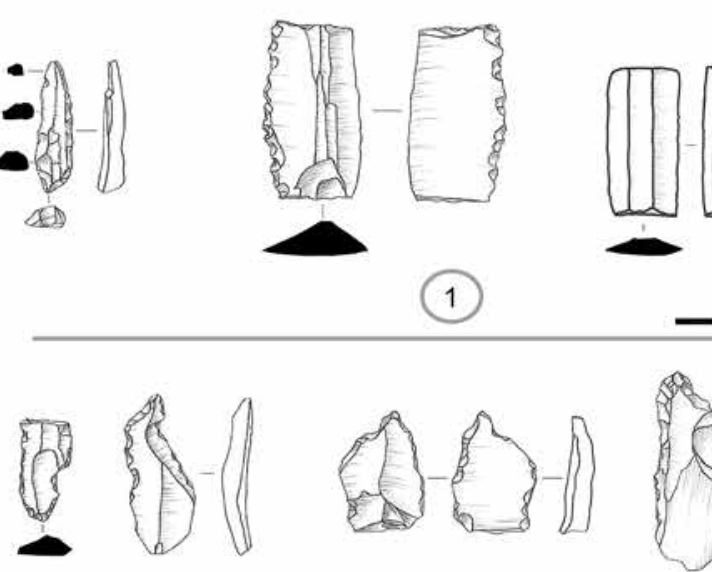

(1)
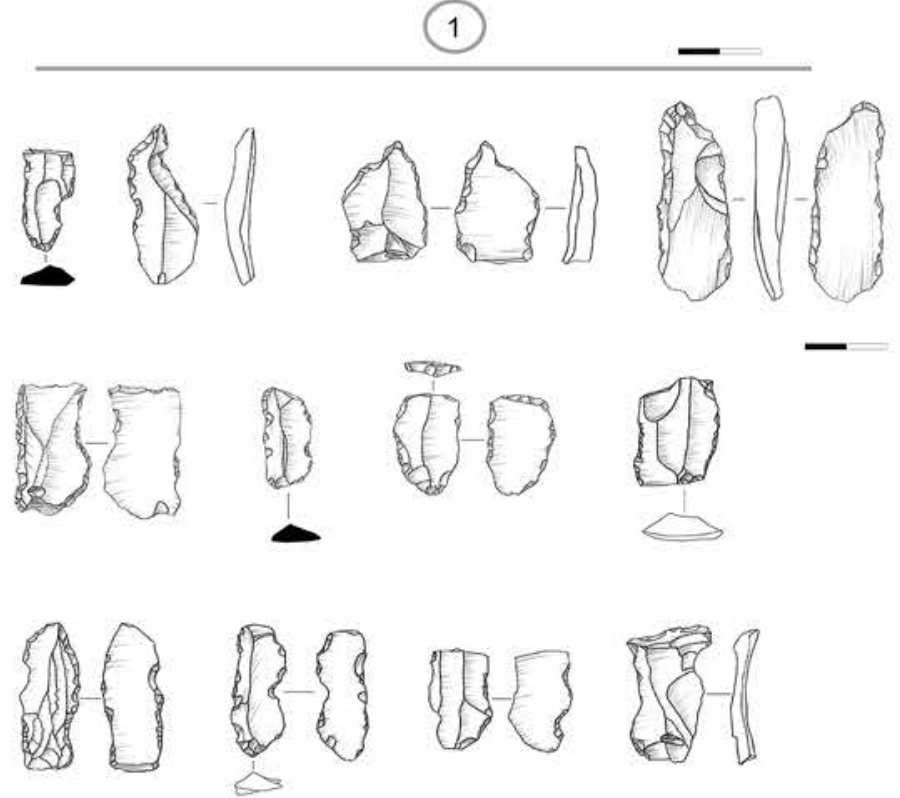

(3)
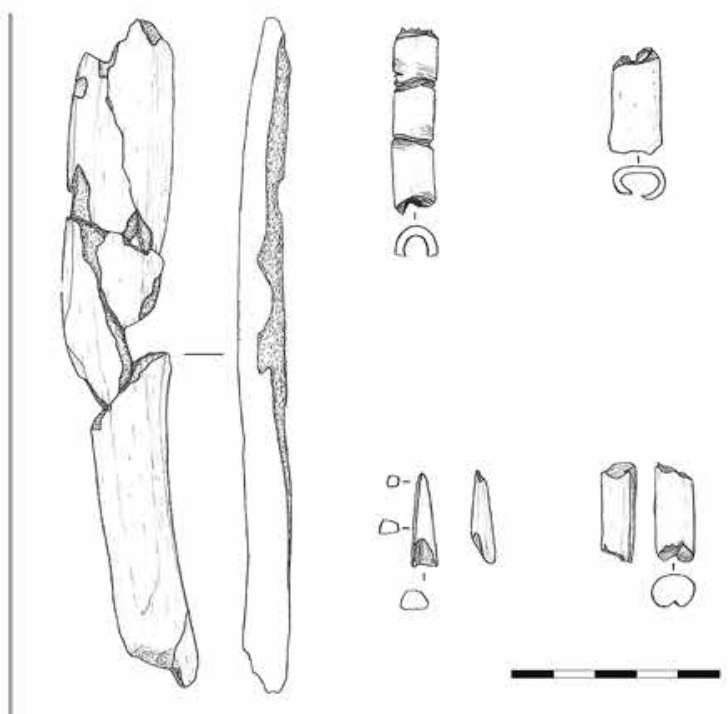

(4)

Fig. 5. Industria lítica y ósea de San Adrian. 1) punta de dorso, elemento de hoz y lámina de sección trapezoidal; 2) raspadores; 3) muescas, denticulados y piezas retocadas; 4) industria ósea: espátula, matrices de cuentas, punzón de hueso, varilla (Dibujos: J. Tapia) / Lithic and bone industries from San Adrian. 1) backed point, sickle piece and trapezoidal blade; 2) endscrapers; 3) notches, denticulates and retouched pieces; 4) bone industry: spatula, bead-matrices, awl, rod (Drawings: J. Tapia). 
También se han hallado dos fragmentos de diáfisis de huesos largos recortados transversalmente mediante aserrado, que interpretamos como soportes para fabricar cuentas cilíndricas, y dos fragmentos de concha de berberecho presumiblemente empleados como elementos de adorno.

\subsection{Restos humanos}

Nuestra intervención ha permitido recuperar 3 nuevos restos humanos que se suman a los hallados en 1984, correspondientes a un individuo infantil y otro adulto. El primero de ellos es una vértebra lumbar (L5) de un individuo masculino adulto joven, hallada en la UE 1202-inferior, y cuya atribución a la ocupación del Bronce es segura; el segundo es un maxilar de un individuo de unos 8 años de edad, recuperado en la UE 1201 y por tanto su adscripción a la Edad del Bronce es probable, pero no segura; y por último, fuera de contexto se recuperaron en el exterior de la cueva dos fragmentos de un mismo cúbito izquierdo, correspondiente a un individuo adulto joven.

\section{5.- DISCUSIÓN Y CONCLUSIONES: LOS MO- DOS DE VIDA DURANTE EL BRONCE ANTIGUO}

Los datos recuperados en San Adrian permiten interpretar este contexto como resultado de una serie de ocupaciones de carácter habitacional desarrolladas a lo largo del Bronce Antiguo (ca. 1900-1500 cal BC). Las estructuras, tanto el posible fondo de cabaña como otras instalaciones complementarias (hogares, silos, etc.), no obedecen a la organización espacial propia de un hábitat permanente, sino que aparecen amortizadas unas por otras. Este comportamiento, opuesto a las tareas de limpieza y mantenimiento que requiere un hábitat estable, apunta a una superposición de ocupaciones temporales en las que se realizan nuevas estructuras sin reaprovechar las preexistentes.

La presencia de numerosos restos de fauna doméstica y de un abundante utillaje de uso común y variado, sugiere el desplazamiento hasta San Adrian de una parte importante del grupo provisto de su bagaje cotidiano de bienes y pertrechos, y permite descartar que se trate de ocupaciones funcionalmente especializadas.

A pesar de tratarse de un sitio de montaña, la recuperación de semillas de trigo carbonizadas, la constatación de un entorno forestal en retroceso por la acción antrópica, y la presencia de utillaje de siega, permiten reivindicar, frente a un modelo predominantemente pastoralista, el importante peso de la agricultura en la base de subsistencia de estos grupos. Más aún, la escasa capacidad de dispersión de los pólenes de Cerealia registrados en los perfiles polínicos permite situar los cultivos a una distancia inferior a $2 \mathrm{~km}$ en torno a San Adrian.
El repertorio artefactual de San Adrian revela la ejecución de tareas de tipo cotidiano y variado. Se constata la producción y uso de utillaje diverso en sílex y hueso, destinado tanto al procesado de recursos animales (carnicería, curtido y cosido de pieles, ¿caza?), como de vegetales (siega), y a la producción de otros bienes (cuentas de hueso, ¿utillaje de madera?). El variado conjunto cerámico, por su parte, muestra la coexistencia de recipientes de diferentes diseños, capacidades y decoraciones que sugieren el desempeño de diversas funciones.

La presencia mayoritaria de materias primas locales, combinada con la incorporación de algunos bienes procedentes de distancias medias (conchas del cantábrico, sílex del Ebro), revela un modelo de explotación de recursos locales complementado por intercambios a media distancia para la obtención de productos de carácter igualmente cotidiano, pero de mayor calidad.

En definitiva, los datos apuntan a un poblamiento estable en el territorio, donde se explota un amplio abanico de recursos locales, y que permite el mantenimiento de intercambios frecuentes con las comunidades vecinas. El hábitat, sin embargo, no refleja asentamientos permanentes, sino que se articula mediante la ocupación de espacios de montaña durante parte del año, combinando los recursos que ofrece este entorno con las tareas de subsistencia ordinarias. Esta movilidad garantizaría tanto el control y acceso a los recursos del territorio, como el mantenimiento de redes de intercambio con grupos vecinos, reforzando el papel de estas comunidades como actores en la circulación de bienes de consumo.

La ausencia de bienes de prestigio -especialmente metálicos- contrasta con la aparición indiferenciada, en el mismo contexto de ocupación que el resto de materiales, de otros objetos igualmente obtenidos mediante intercambio (láminas de sílex, conchas), así como de restos frecuentes en contextos simbólicos o funerarios -cuentas de hueso, cerámicas cordadas, o los mismos restos humanos-.

Buena parte de estas características son extensibles al cercano yacimiento de Haltzerreka, tanto en lo relativo a la proximidad entre áreas de hábitat y funerarias, como a la presencia de materiales del Ebro, del área cantábrica, y continentales (sílex del Flysch, de Urbasa y de Chalosse) (MUJIKA ET AL., 2009). También la imposibilidad de identificar estructuras en Haltzerreka podría deberse a su carácter de hábitat no permanente, e igualmente se han recuperado materiales de cierto valor asociados normalmente a contextos funerarios, como el ámbar.

Los caracteres técnicos y decorativos de ambos conjuntos cerámicos demuestran la existencia de influencias pirenaicas y continentales, lo que refuerza el papel transmisor de estos grupos en la circulación de bienes e ideas entre el interior peninsular, la cuenca aquitana, y la región cantábrica. 
De este modo, puede plantearse la existencia de un patrón de ocupación del territorio basado en el desplazamiento periódico de todo el grupo a zonas de montaña, destinado tanto a realizar tareas de subsistencia comunes como a favorecer contactos e intercambios con comunidades vecinas. Estos intercambios permiten el acceso a otros bienes cotidianos de procedencia peninsular y continental, y se distribuyen en el seno de los grupos humanos sin aparentes restricciones de rango social. Sin embargo, tanto la ausencia de objetos metálicos en estos contextos cotidianos, como la necesidad de una planificación de las estrategias de subsistencia, de control territorial y de interlocución en los intercambios de bienes, sugieren la existencia de unas élites que reivindican su posición mediante el monopolio de los objetos de mayor coste y significado, como las armas metálicas (BRADLEY, 1990).

Los resultados obtenidos en San Adrian permiten estudiar las sociedades del Bronce Antiguo del cantábrico desde una nueva perspectiva, distinta de su tradicional caracterización como comunidades ganaderas marginales. Al contrario, el tipo de hábitat y la gestión del territorio plantean un escenario donde se reivindica el papel de estas comunidades agropecuarias como transmisor de influencias y bienes entre Europa y la Península Ibérica, y relega las restricciones en el acceso a bienes de prestigio a los elementos de mayor coste y significado simbólico como las armas y objetos metálicos.

\section{AGRADECIMIENTOS}

El proyecto de intervención arqueológica ha contado con financiación de la Diputación Foral de Gipuzkoa, la Parzonería General de Gipuzkoa y Álava, y el Ayuntamiento de Zegama.

\section{REFERENCIAS}

\section{ALDAY RUIZ, A.}

1992 Síntesis sobre la secuencia cultural Neolítico-Edad del Bronce en el País Vasco. Sancho el sabio: Revista de cultura e investigación vasca, 2, 19-50.

ALTUNA, J., MARIEZKURRENA, K., ARMENDARIZ, A., DEL BARRIO, L., UGALDE, T. \& PEÑALVER, J.

1982 Carta Arqueológica de Guipúzcoa. Munibe 34, 1-4.

ALTUNA, J.; ARMENDARIZ, A.; ETXEBERRIA, F.; MARIEZKURRENA, K.; PENNALVER, X. \& ZÜMALABE, F.

1995 Gipuzkoa. Karta Arkeologikoa. Carta Arqueológica. II. Haitzuloak-Cuevas. Munibe (Antropologia-Arkeologia), Supl. $n^{\circ}$ 10. Donostia-San Sebastián.

APELLÁNIZ, J. M.

1973 Corpus de materiales de las culturas prehistóricas con cerámica de la población de Cavernas del País Vasco Meridional. Munibe, suplemento $n^{\circ} 1$

\section{ARMENDARIZ, A.}

1984 Dos nuevas hachas de metal en Guipúzcoa. Munibe 36, 67-69.

1990 Las cuevas sepulcrales en el País Vasco. Munibe (Antropologia-Arkeologia) 42, 153-160

\section{ARMENDARIZ, A. \& ETXEBERRIA, F.}

1983 Las cuevas sepulcrales de la Edad del Bronce en Guipúzcoa. Munibe 35, 247-354.

BRADLEY, $R$.

1990 The Passage of Arms. Cambridge University Press. Cambridge

BRONK RAMSEY, C

2001 Development of the radiocarbon calibration program. Radiocarbon, 43, 355-363.

2009 Bayesian analysis of radiocarbon dates. Radiocarbon, 51, 337-360.

HARRIS, E. C.

1991 Principios de Estratigrafía Arqueológica. Crítica. Barcelona.

MUJIKA, J. A. \& EDESO, J. M.

2011 Lehenengo nekazari-abeltzainak Gipuzkoan. Neolitotik Burdin Arora. Los primeros agricultores y ganaderos en Gipuzkoa. Del Neolítico a la Edad del Hierro. Donostia: Gipuzkoako Foru Aldundia (Arkeologia 0.2.)

MUJIKA, J. A.; PEÑALVER, X. \& TARRIÑO, A.

2009 Haltzerreka (Gipuzkoa): un asentamiento del bronce antiguo al aire libre en un medio de montaña. Kobie Paleoantropologia 28, 89-120.

REIMER, P. J., BARD, E., BAYLISS, A., BECK, C. W., BLACKWELL, P. G., BRONK RAMSEY, C., BUCK, C. E., CHENG, H., EDWARDS, R. L., FRIEDRICH, M., GROOTES, P. M., GUILDERSON, T. P., HAFLIDASON, H., HAJDAS, I., HATTÉ, C., HEATON, T. J., HOFFMANN, D. L., HOGG, A. G., HUGHEN, K. A., KAISER, K. F., KROMER, B., MANNING, S., NIU, M., REIMER, R. W. RICHARDS, D. A. MARIAN SCOTT, E., SOUTHON, J. R., STAFF, R. A., TURNEY, C. S. M. \& VAN DER PLICHT, J.

2013 INTCAL13 and Marine 13 radiocarbon age calibration curves $0-50,000$ years cal BC. Radiocarbon, 55, 1869 1887.

RUIZ ALONSO, M.

2009 Aprovechamiento de los recursos vegetales en la Edad del Bronce: el yacimiento de Haltzerreka (Gipuzkoa, País Vasco). Kobie Paleoantropologia 28, 121-130.

STRAUS, L. G. \& GONZÁLEZ MORALES, M. R.

2012 El Mirón Cave, Cantabria Spain. The Site and Its Holocene Archaeological Record. Alburquerque: University of New Mexico Press. 